\title{
'Referral into a void': opinions of general practitioners and others on single point of access to mental health care
}

\author{
Rosalind Raine MBBS PhD ${ }^{1}$ Simon Carter PhD ${ }^{1} \quad$ Tom Sensky MD ${ }^{2} \quad$ Nick Black MD FFPH ${ }^{1}$
}

J R Soc Med 2005;98:153-157

\section{SUMMARY}

In the past, British general practitioners (GPs) have referred patients to individual consultants. There is now a trend towards generic referral, whereby the specialist team is consulted via a single point of access. We examined the impact of this innovation on the relationship between GPs and specialists in mental health care. Sixteen groups of randomly selected GPs and mental health professionals (MHPs) in England discussed clinical scenarios involving the use of mental health interventions for patients with functional somatic symptoms. The meetings were audiotaped, transcribed and analysed to the point at which no major new themes were emerging. The final analysis was confined to a purposive sample of six of the sixteen groups, comprising 54 GPs and 15 MHPs.

Although the rationale for the single point of access was clear to both GPs and MHPs, the approach erected boundaries because it impeded the establishment of professional relationships and transfer of knowledge. GPs thought the system reduced their capacity to be accountable to their patients and limited the potential for their own professional development. MHPs did not seem to be aware of GPs' concerns.

Effective interprofessional management of individual patients depends upon confidence in colleagues' skills and good communication. Factors that hamper these must be addressed in the development of this system of referral.

\section{INTRODUCTION}

Health services comprise an array of semi-autonomous professionals in loose networks. ${ }^{1}$ From the patients' perspective, the networks should be seamless, and traditional interprofessional relationships are increasingly being challenged. One innovation that accords with multiprofessional teamworking, and the requirement to redesign care around patients, is single-point-of-access referral - a departure from the traditional arrangement whereby general practitioners (GPs) refer their patients to individual specialists. The idea is to avoid the inefficiencies that arise from use of different referral forms, different points of access, different assessment tools and multiple client records. The health professional who receives the referral is expected to have full information about the range of services available and take responsibility for assigning the patient to the appropriate person. This approach has been

1Department of Public Health and Policy, London School of Hygiene \& Tropical Medicine, Keppel Street, London WC1E 7HT; ${ }^{2}$ Department of Psychological Medicine, Imperial College of Science, Technology and Medicine, West Middlesex University Hospital, Isleworth, Middlesex TW7 6AF, UK

Correspondence to: Dr R Raine

E-mail: rosalind.raine@Ishtm.ac.uk developed in several areas, including primary care, emergency care, health and social rehabilitation, and outpatient mental health services.

For the single-point-of-access system to work, practitioners need to have confidence in the process and a clear understanding of each others' roles and skills. In the psychiatric sector, even traditional referral systems have given rise to complaints from primary care about poor communications and exclusion from planning, ${ }^{2-6}$ with possible adverse effects on referral rates $;^{5}$ and psychiatrists themselves recognize the existence of communication difficulties. ${ }^{7}$ In the present study we explored the attitudes of English GPs to single-point-of-access referral and, in addition, the perceptions of mental health professionals (MHPs) on this matter.

\section{METHODS}

The research programme has been described in detail elsewhere. ${ }^{8}$ Briefly, national random samples of GPs and MHPs were selected from the Department of Health GP database for England ( $N=27723)$, the Royal College of Psychiatrists liaison section database and the British Association of Behavioural and Cognitive Psychotherapists 
database (total $N=720$ ). We established sixteen groups involving 135 GPs and 42 MHPs between February 2002 and January 2003. Each group met for a facilitated discussion about the appropriateness of mental health interventions for patients with three conditions that commonly present with physical symptoms but in which psychological factors may be prominent - chronic fatigue syndrome, irritable bowel syndrome and chronic back pain. Each meeting lasted about four hours and was audiotaped and later transcribed verbatim. The meetings were all held at the same venue and followed a written protocol. A purposive sample of six of the sixteen groups, comprising a total of 54 GPs and 15 MHPs, was selected for the analysis presented here; three consisted of GPs only $(n=10, n=10$; $n=13)$ and three had both GPs and MHPs $(n=7+6, n=7+5$, $n=7+4)$.

The initial transcripts and 'journal' notes were examined independently by two of the authors to draw up a preliminary list of themes. The authors then met to compare and discuss identified themes, which were used iteratively and applied to later data. We gave specific attention to comments about the single-point-of-entry system, mental health interventions and interprofessional relations. We maintained constant vigilance for statements that might conflict with the emerging thematic and conceptual relations. By the time the transcripts of six groups had been analysed no major new themes were emerging and we judged that 'theoretical saturation' had been reached.

The study was approved by the ethics committee of the London School of Hygiene and Tropical Medicine.

\section{RESULTS}

Both GPs and MHPs believed the rationale for the approach to be that GPs cannot be expected to know which therapy will be most appropriate. Some GPs had no argument with this approach because they had scant knowledge of the various therapies and lacked the personal information on individual MHPs that might influence their choice of specialist. MHPs favoured the single-point-of-access system because choice of therapy demands expert patient assessment.

\section{Opinions of GPs}

Most of the comments from GPs, reflected reluctant acceptance at best, and these views are summarized under three headings.

\section{The approach hinders mutual understanding}

A single-point-of-access system necessitates referral such a team's competence to decide what was best:

'Would they agree amongst themselves as to the best way of approaching some of these chronic illnesses? I am not sure they would' (GP77).

Such comments reflected unease about dealing with non-medical colleagues whose culture and language were not those of medicine. Some GPs expressed favourable opinions about non-medical MHPs and criticized GP colleagues for professional arrogance:

[The feeling that] 'these people are not quite professionals pervades the whole of medicine' (GP80).

Negative comments on the status of psychologists and other mental health professionals were confined to the GPonly groups:

'They have to have very high powered degrees . . . to preserve the rarity of their jobs ... they want to be special . . . it's absolute nonsense' (GP7);

'Our patients are seen by a case worker. God knows what they are' (GP81).

GPs expressed a spectrum of attitudes towards mental health interventions. Enthusiasm was expressed by GPs when patients reported positive experiences or when GPs empathized with the underlying principles

'I've had a lot of positive feedback' (GP42);

'I'm interested in behaviour therapy because it's about coping with your body' (GP78);

'If [patients] have a misunderstanding about the nature of the disease, then looking at cognitions might make a difference' (GP48).

Knowledge of research findings produced a mixed reaction depending on GPs' views of the evidence. Some felt compelled to accept the evidence:

'We have been bombarded by stuff saying that CBT [cognitive behaviour therapy] is the best thing since sliced bread' (GP102).

Others questioned the relevance of research findings to their practice:

'The evidence base is very poor . . . there were very small numbers' (GP10) 
'General practice depression isn't the same as depression in psychiatry' (GP7)

'The evidence is not from general practice' (GP9)

'Any study could show an effect even if it's a very slight effect and that might not be all that clinically helpful' (GP114).

Grudging acceptance reflected the belief that either the rationale behind interventions could be explained in a way that would be acceptable to patients, or that there was a lack of alternatives, or that the interventions were a means of getting rid of the problem:

'You can always sell them behaviour therapy on the basis that relaxation helps to relax their muscles. They are more likely to go for it than for having their heads shrunk' (GP80);

'We don't know any better treatment than this' (GP101);

'It gets patients out of our hair' (GP10);

'It is an alternative to saying "there is nothing further to be done", reflecting a willingness to keep trying to do something' (GP9)

Adverse comments sometimes reflected a lack of experience of the benefits of mental health interventions because these were not available locally, a lack of conviction about patient adherence and thus treatment effectiveness, or concern about possible harm from psychotherapy

'If you haven't got these treatment options you are going to be sceptical about using them' (GP10);

'These techniques depend very highly on doing . . . homework. If patients are not doing homework, efficacy is reduced' (GP98);

'You have to ask, is there an addictive potential?' (GP101).

These GPs doubted the difference between interventions or questioned their scientific legitimacy or even ridiculed them:

'They all do more or less the same thing' (GP3);

'It might be the throwing of bones or the reading of prunes for all we know' (GP80);

'How do you re-train a bowel? Down boy, down!' (GP44).
Such scepticism was not helped by the perceived anonymous nature of the single-point-of-access, which was not organized to allow much dialogue between professionals:

'You get "this patient has completed a course of assessment"; you don't get their opinion or where to go from there' (GP77).

Interprofessional communication was also hampered by a reluctance of GPs to admit ignorance to non-medical colleagues:

'You feel you can write to people in other [medical] specialties saying "I'm afraid I have no idea what this means". But I would not do that to a [non-medical] psychotherapist because I might seem ignorant' (GP81).

These communication barriers also reduced the potential for MHPs to appreciate the reasons for GPs' treatment decisions. Thus GPs' use of antidepressants was dismissed by MHPs as thoughtless, reinforcing patients' beliefs, or as an easy option:

'The Pavlovian response "oh you are depressed, let's give you antidepressants", (MHP42);

'You give them [a tablet] that fits in with the way they see things' (MHP39);

'[easier] than persuading [the patient] that it might be psychological' (MHP29).

\section{The approach undermines GPs' relationships with their patients}

The anonymous nature of the single-point-of-access system was perceived to undermine GPs' credibility and, by extension, their relationship with patients:

"When the patient asks "why are you sending me there and what is going to happen to me?", if you have no idea, that could be a bit of a problem' (GP116).

Second, GPs' ideology of patient protection was breached:

'I would like to make the choice . . . in the best interests for my patient. If I don't know whether they are going to see Dr A or Dr B, then I would feel that I'm giving my patients a worse service' (GP82). 
This contrasted with referrals to other specialties where there was some personal knowledge of the clinician to whom the patient would be referred:

'For the rest of our secondary referrals we know that the patient will do better with this consultant because he is more direct, and this one is much more reflective' (GP88).

However, the idea that GPs retain more control in referrals which do not use the single point of access was disputed by other participants:

'If we send someone to a rheumatologist, we don't say "I want you to give him methotrexate". You trust that guy will choose whatever is the most appropriate treatment (GP79).

\section{The approach hampers GPs' efforts to improve their knowledge and skills}

GPs said that the lack of effective communication impeded their professional development because they were not provided with information that would enable them to learn from their patients' experiences. They were also unable to supplement the therapy provided:

'We have got to work in harmony with them and I would want to know what particular discipline is being used because I would want to try and mirror it' (GP87).

Finally, because the approach eliminated the need to choose an appropriate therapy for patients, it was blamed for perpetuating GPs' commonly stated lack of knowledge about mental health interventions:

'The single point of entry encourages you to be quite lazy . . . and not really to apply your mind as to what sort of therapy might be useful' (GP84).

\section{Mental health professionals' perceptions}

MHPs did not make explicit comments that demonstrated awareness of GPs' concerns about single-point-of-access referral; they did seem aware of the fellowship between doctors but not of the potential implications of disrupting these relationships. It was assumed that GPs took a pragmatic approach towards referral:

'In the olden days, the GP would specially want the patient to be seen by a doctor. Now we have moved away from that. [Patients] just get referred to a team on a rota basis' (MHP39).

There was some discussion of an alternative system in which MHPs worked in GP surgeries; this, however, centred on the advantages for patients (that it destigmatized psychological referral) and ignored the potential for improving interprofessional communication. Though we have no data on the matter, we speculate that work pressures caused MHPs to focus on their own needs and to neglect those of GPs.

\section{DISCUSSION}

This qualitative study provides evidence that English GPs have deep misgivings about single-point-of-access referral to mental health care in terms of professional communication, accountability to patients and their own professional development. Before drawing firm conclusions, however, we must address the limitations of the study. First, it included all-GP groups and mixed GP/MHP groups but no all-MHP groups. (We noted that unfavourable comments about MHPs were not made in the mixed groups.) More data were available from GPs than from MHPs and this may mean that our results are more generalizable for GPs. Second, the protocol was not designed to explore proactively the reasons for participants' beliefs. It is therefore possible that we have not fully uncovered all the explanations for our findings. For example, the conclusion that the MHPs did not seem aware that GPs had reservations about single-point-of-access referral might or might not have been modified if we had been able to ask participants to explore the issue. Also our method only allowed us to analyse what participants said they do, not what they actually did in practice. Third, our findings may not be generalizable to professional relationships between GPs and specialists in other fields. It could be argued that boundaries between GPs and MHPs are likely to be particularly high in respect of somatic conditions such as those discussed here, where neither GP nor patient may be keen on MHP involvement. On the other hand, one might expect MHPs working with these conditions to be sensitive to the need for interprofessional collaboration.

This is not the first British study to examine the views of GPs on referral of psychiatric patients 'into a void'. Puri and co-workers in London found that most of the GPs who refrained from using an open-access emergency service did so because they wanted their patients to be seen by a consultant. ${ }^{9}$ Although single-point-of-access referral to specialist services is clearly beneficial in many ways, we find that in mental health care it can erect barriers between GPs and specialists. GPs expressed doubt about serving the interests of their patients if they did not know which specialist would make the assessment, the content and value 
of the interventions offered, or what options were likely to follow. The single-point-of-access system was also perceived to make it more difficult for GPs and MHPs to acquire an understanding of each other's work. These issues need to be considered in the development of clinical services and in the training of healthcare professionals.

Acknowledgments We thank the GPs and mental health specialists who participated in the study; Judy Green for providing expert advice and for commenting on drafts; Kirsten Larkin for formatting the questionnaire, providing administrative support to Rosalind Raine (RR), recording the meetings and transcribing the data; and the Medical Research Council (MRC) for funding a Clinical Scientist Fellowship for RR. The MRC, which was the sole funding source, had no involvement in the study design or conduct, or in the writing of the paper.

The study received ethnical approval from the London School of Hygiene and Tropical Medicine Ethics Committee.

\section{REFERENCES}

1 Griffiths L. Making connections: studies of the social organisation of healthcare. Sociol Health Illn 2003;25:155-71

2 Little D, Hammond C, Kollisch D, Stern B, Gagne R, Dietrich A. Referrals for depression by primary care physicians. J Fam Pract 1998;47:375-7

3 Orleans T, George L, Houpt J, Brodie K. How primary care physicians treat psychiatric disorder: A national survey of family practitioners. $A m J$ Psychiatry $1985 ; 142: 52-7$

4 Falloon I, Ng B, Bensemann C, Kydd R. The role of general practitioners in mental health care: a survey of needs and problems. $N Z$ Med J 1996;109:34-6

5 Brown J, Weston W. A survey of residency-trained family physicians and their referral of psychosocial problems. Fam Med 1992;24:193-6

6 Adeyemi J, Olonade P, Amira C. Attitude to psychiatric referral: a study of primary care physicians. Niger Post Med J 2002;9:53-8

7 Tanielian T, Pincus H, Dietrich A, et al. Referrals to psychiatrists. Assessing the communication interface between psychiatry and primary care. Psychosomatics 2000;41:245-52

8 Raine R, Sanderson C, Hutchings A, Carter S, Larkin K, Black N. An experimental study of determinants of group judgments in clinical guideline development. Lancet 2004;364:429-37

9 Puri BK, Hall AD, Reefat R, Mayer K, Tyrer P. General practitioners' views on an open referral system to a community mental health service. Acta Psychiatr Scand 1996;94:133-6 